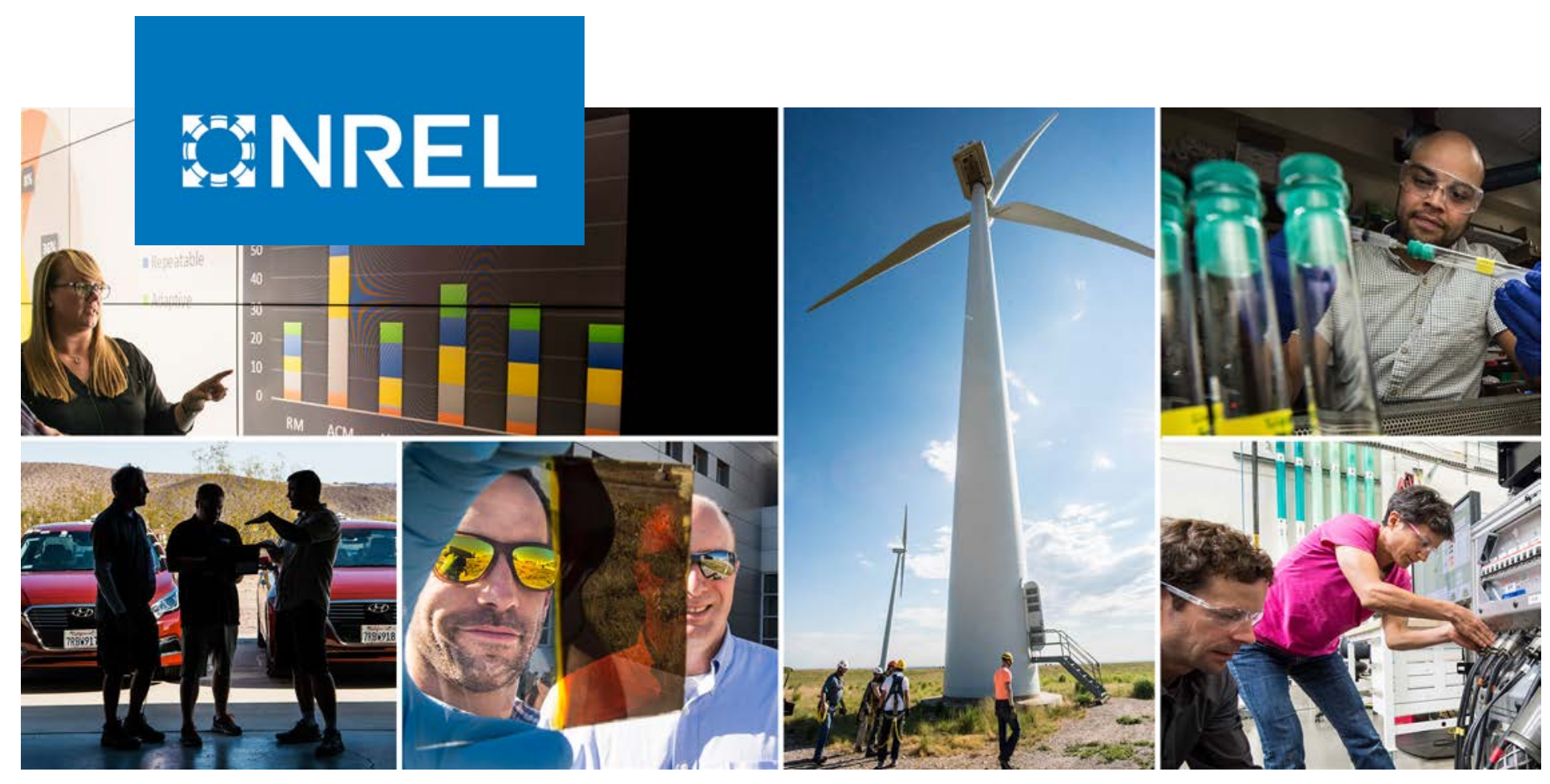

\title{
Impedance-Based Characterization of Power System Frequency Response
}

\section{Preprint}

Shahil Shah and Vahan Gevorgian

National Renewable Energy Laboratory

Presented at the 2019 IEEE Power and Energy Society General Meeting (IEEE PES GM) Atlanta, Georgia August 4-8, 2019

NREL is a national laboratory of the U.S. Department of Energy Office of Energy Efficiency \& Renewable Energy

Operated by the Alliance for Sustainable Energy, LLC

This report is available at no cost from the National Renewable Energy Laboratory (NREL) at www.nrel.gov/publications.

\section{Conference Paper}

NREL/CP-5D00-72478

October 2019 


\section{FANREL}

\section{Impedance-Based Characterization of Power System Frequency Response}

\section{Preprint}

\section{Shahil Shah and Vahan Gevorgian}

National Renewable Energy Laboratory

\section{Suggested Citation}

Shah, Shahil, and Vahan Gevorgian. 2019. Impedance-Based Characterization of Power System Frequency Response: Preprint. Golden, CO: National Renewable Energy Laboratory. NREL/CP-5D00-72478. https://www.nrel.gov/docs/fy20osti/72478.pdf.

(C) 2019 IEEE. Personal use of this material is permitted. Permission from IEEE must be obtained for all other uses, in any current or future media, including reprinting/republishing this material for advertising or promotional purposes, creating new collective works, for resale or redistribution to servers or lists, or reuse of any copyrighted component of this work in other works.

NREL is a national laboratory of the U.S. Department of Energy Office of Energy Efficiency \& Renewable Energy Operated by the Alliance for Sustainable Energy, LLC

This report is available at no cost from the National Renewable Energy Laboratory (NREL) at www.nrel.gov/publications.

Contract No. DE-AC36-08G028308
Conference Paper

NREL/CP-5D00-72478

October 2019

National Renewable Energy Laboratory 15013 Denver West Parkway Golden, CO 80401

303-275-3000 • www.nrel.gov 


\section{NOTICE}

This work was authored by the National Renewable Energy Laboratory, operated by Alliance for Sustainable Energy, LLC, for the U.S. Department of Energy (DOE) under Contract No. DE-AC36-08GO28308. Funding provided by the U.S. Department of Energy Office of Energy Efficiency and Renewable Energy Wind Energy Technologies Office. The views expressed herein do not necessarily represent the views of the DOE or the U.S. Government. The U.S. Government retains and the publisher, by accepting the article for publication, acknowledges that the U.S. Government retains a nonexclusive, paid-up, irrevocable, worldwide license to publish or reproduce the published form of this work, or allow others to do so, for U.S. Government purposes.

This report is available at no cost from the National Renewable Energy Laboratory (NREL) at www.nrel.gov/publications.

U.S. Department of Energy (DOE) reports produced after 1991 and a growing number of pre-1991 documents are available free via www.OSTI.gov.

Cover Photos by Dennis Schroeder: (clockwise, left to right) NREL 51934, NREL 45897, NREL 42160, NREL 45891, NREL 48097, NREL 46526 .

NREL prints on paper that contains recycled content. 


\title{
Impedance-Based Characterization of Power System Frequency Response
}

\author{
Shahil Shah and Vahan Gevorgian \\ National Renewable Energy Laboratory (NREL), Golden, CO 80401, USA \\ Email: Shahil.Shah@nrel.gov, Vahan.Gevorgian@nrel.gov
}

\begin{abstract}
This paper presents an impedance-based noninvasive method for the characterization of power system frequency response in real time in the absence of a transient event. The proposed method measures transfer function from the injected active power to the frequency at the point of interconnection for the estimation of system inertia, primary frequency response, and the speed of the primary frequency control of the system. The socalled frequency response transfer function $F R(s)$ is measured by injecting perturbations in the active power output of a battery energy storage system. The measured response of $F R(s)$ can also predict the frequency nadir and ROCOF following a transient event. The relationship between $F R(s)$ and the system impedance response as seen from the point of interconnection is also derived in this paper; it can be used for the control design of frequency support services by inverter-coupled generation and storage. The proposed methodology is demonstrated on a modified IEEE 9-bus system with $25 \%$ penetration of wind and PV generation.
\end{abstract}

Index Terms-Impedance-based analysis, inertia, primary frequency response, ROCOF, frequency adequacy.

\section{INTRODUCTION}

The security and resilience implications of operating lowinertia power systems require the development of new real-time tools for the analysis of frequency response adequacy so that system operators can ensure system frequency stability under any conceivable contingency and for any resource dispatch scenarios [1]. Unlike traditional statistical approaches to frequency response adequacy estimation [2]-[4], the method proposed in this paper has a multi-pronged impact. It is capable of identifying system security issues arising from generation mixes in real time at the beginning of any security-constrained unit dispatch interval while simultaneously identifying other potential resonance and stability problems that inverter-coupled energy storage and renewable generation can help mitigate. The method allows for the conduction of essentially a fundamental frequency response adequacy evaluation in real-time, a capability that has never been in existence within the energy industry.

Frequency response characteristics following a transient event including frequency nadir, rate of change of frequency (ROCOF),

This work was authored by Alliance for Sustainable Energy, LLC, the manager and operator of the National Renewable Energy Laboratory for the U.S. Department of Energy (DOE) under Contract No. DE-AC36-08GO28308. Funding provided by U.S. Department of Energy Office of Energy Efficiency and Renewable Energy Wind Energy Technologies Office. The views expressed in the article do not necessarily represent the views of the DOE or the U.S. Government. The U.S. Government retains and the publisher, by accepting the article for publication, acknowledges that the U.S. Government retains a nonexclusive, paid-up, irrevocable, worldwide license to publish or reproduce the published form of this work, or allow others to do so, for U.S. Government purposes. and settling frequency - depend on system inertia and the primary frequency response (PFR) of generators and loads [2]. The PFR of an interconnection is typically measured in $\mathrm{MW} / 0.1 \mathrm{~Hz}$; it shows the amount of power disturbance that will result in the change in frequency by $0.1 \mathrm{~Hz}$ during steady state following an event. Several studies have shown that both inertia and PFR are gradually declining in many power systems around the world, primarily because of the increasing penetration of power electronics-coupled renewable generation and the displacement of conventional generation [3], [4]. This limits the penetration level of renewable generation an interconnection can absorb without causing reliability concerns.

The Federal Energy Regulatory Commission recently introduced the BAL-003-1 standard, which requires each balancing authority within an interconnection to maintain a minimum PFR depending on its share of generation in the interconnection [5]. System inertia and PFR are periodically measured in U.S. interconnections using statistical methods based on the responses during transient events [6]. Using these methods, it is not possible to characterize the frequency response in real time for a specific security-constrained dispatch scenario. The methods also do not provide insights on the role of frequency support from energy storage and renewable generation, and the effect of the location of energy storage used for providing frequency support. Hence, the design of frequency support by renewable generation and storage has relied on numerical simulations with a high degree of simplifications [6], [7].

In this paper, we present an impedance-based noninvasive approach for the characterization of power system frequency response in real time in the absence of a transient event. The proposed method reveals the frequency response of the system using measurements at the point of interconnection. It also provides an analytical basis for the control development of frequency support by renewable generation and storage. The method is demonstrated on a modified IEEE 9-bus system. Estimation of system inertia, PFR, and the speed of primary frequency control is demonstrated using the proposed method. The paper also shows relation between the impedance of the network as seen from the point of interconnection and the frequency response behavior of a network.

\section{FREQUENCY RESPONSE CHARACTERIZATION}

The proposed impedance-based characterization method measures transfer function response from the active power injected at the point of common coupling (PCC) to the measured frequency at the PCC. This requires injection of active power with 


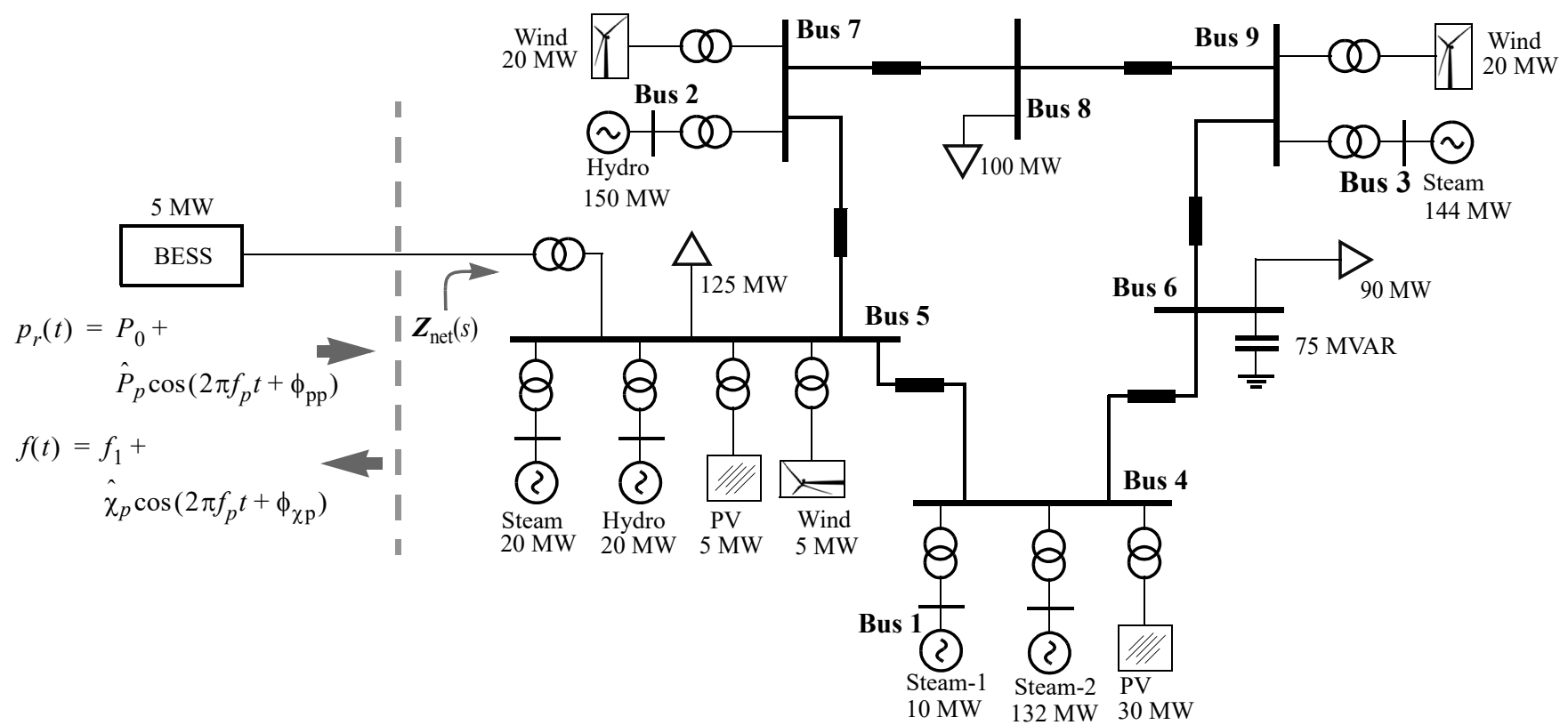

Fig. 1. Simulated modified IEEE 9-bus system.

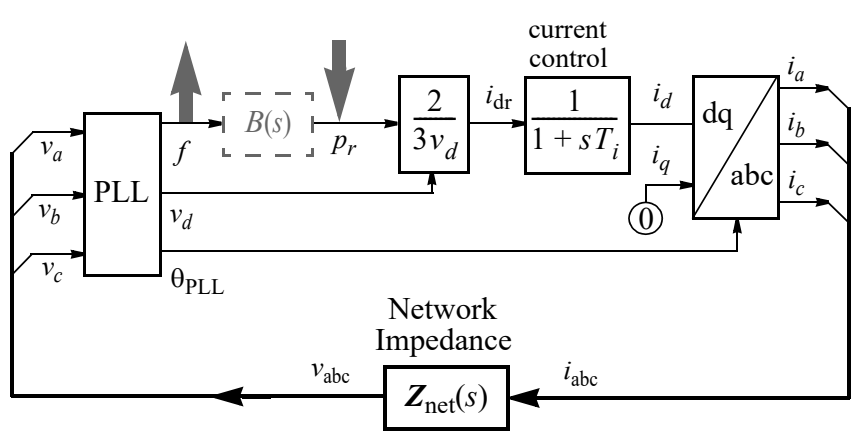

a)

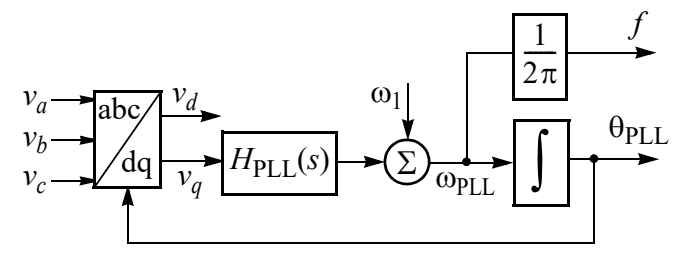

b)

Fig. 2. Active power injection using BESS: a) implementation and b) PLL.

sinusoidal perturbations at different frequencies. Fig. 1 shows modified IEEE 9-bus system used to demonstrate the frequency response characterization method. The inverter-coupled battery energy storage system (BESS) at Bus 5 is used for the injection of active power perturbations. Note that other devices - such as wind turbines, PV inverters, and HVDC converters - can also be programmed to inject perturbations.

Fig. 2 shows the implementation of the proposed method. Sinusoidal perturbation is injected in the reference for the active power, $p_{r}$, supplied by the battery:

$$
p_{r}(t)=P_{0}+\hat{P}_{p} \cos \left(2 \pi f_{p} t+\phi_{\mathrm{pp}}\right)
$$

TABLE I CONVENTIONAL GENERATION: RATINGS, ACTIVE POWER OUTPUT, INERTIA CONSTANT, AND DROOP CONSTANT

\begin{tabular}{c|c|c|c|c}
\hline Generator & $\begin{array}{c}\text { Rating, } \\
S \text { (MVA) }\end{array}$ & $\begin{array}{c}\text { Active } \\
\text { power out- } \\
\text { put in MW }\end{array}$ & $\begin{array}{c}\text { Inertia } \\
\text { constant, } \\
H(\mathrm{~s})\end{array}$ & $\begin{array}{c}\text { Nominal } \\
\text { droop con- } \\
\text { stant }\left(R_{p}\right)\end{array}$ \\
\hline Hydro @ Bus-7 & 150 & 59.18 & 6.0 & 0.05 \\
\hline Hydro @ Bus-5 & 20 & 10.48 & 6.0 & 0.05 \\
\hline Steam @ Bus-5 & 20 & 11.49 & 3.12 & 0.20 \\
\hline Steam-1 @ Bus-4 & 10 & 5.70 & 3.12 & 0.20 \\
\hline Steam-2 @Bus-4 & 132 & 75.78 & 3.12 & 0.20 \\
\hline Steam @ Bus-9 & 144 & 82.66 & 3.12 & 0.20 \\
\hline Total & 476 & 245.29 & $4.15 \mathrm{~s}$ & - \\
\hline
\end{tabular}

where $P_{0}$ is the steady-state active power output of the battery, $\hat{P}_{p}$ is the amplitude of the sinusoidal perturbation, and $f_{p}$ is the perturbation frequency. The d-axis current reference, $i_{\mathrm{dr}}$, is derived from $p_{r}$ and the d-axis component, $v_{d}$, of the voltages $v_{\mathrm{abc}}$ at the PCC. Because the voltage source converter (VSC) current control dynamics are much faster than the frequency response dynamics of a power system, they are represented simply by a low-pass filter with the time constant $T_{i}$ of $4 \mathrm{~ms}$. The BESS output currents $i_{\mathrm{abc}}$ enter the modified IEEE 9-bus system and depending on the network impedance $\boldsymbol{Z}_{\text {net }}(s)$, the perturbations in $i_{\text {abc }}$ result in the perturbations in $v_{\text {abc }}$. As shown in Fig. 2b), a three-phase PLL obtains the frequency measurement $f$, voltage angle $\theta_{\mathrm{PLL}}$, and $v_{d}$.

Under perturbation, the measured frequency, $f$, at the PCC can be represented as:

$$
f(t)=f_{1}+\hat{\chi}_{p} \cos \left(2 \pi f_{p} t+\phi_{\chi \mathrm{p}}\right)
$$

where $\hat{\chi}_{p}$ and $\phi_{\chi \mathrm{p}}$ are, respectively, the amplitude and phase of 

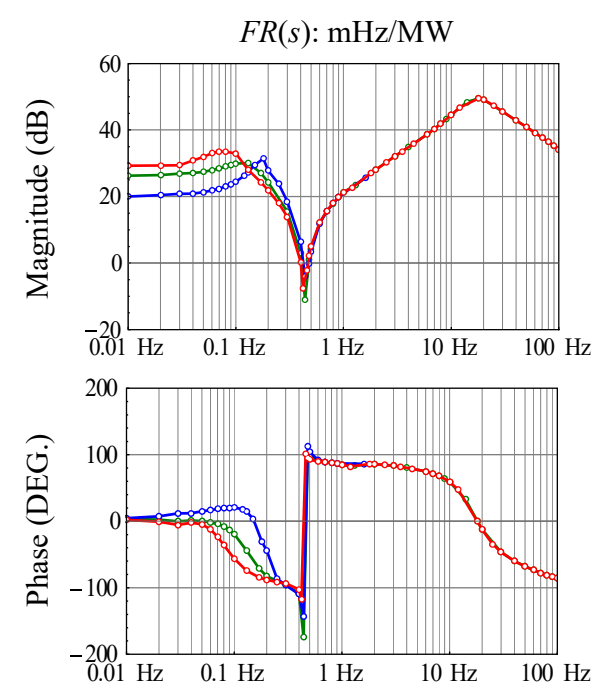

Fig. 3. Measurements of the frequency response transfer function $F R(s)$ for different droop settings in the steam generators of the modified IEEE 9-bus system: a) $20 \%$ droop: red lines, b) 10\% droop: green lines, and c) 5\% droop: blue lines.

the perturbation component in $f$ at frequency $f_{p}$.

The desired frequency response function, $F R(s)$, is defined as:

$$
F R(s) \equiv \frac{\mathbf{F}\left[f_{p}\right]}{\mathbf{P}_{r}\left[f_{p}\right]}
$$

where $\quad s=j 2 \pi f_{p}, \quad \mathbf{F}\left[f_{p}\right]=\left(\hat{\chi}_{p} / 2\right) \exp \left(j \cdot \phi_{\chi \mathrm{p}}\right), \quad$ and $\mathbf{P}_{r}\left[f_{p}\right]=\left(\hat{P}_{p} / 2\right) \exp \left(j \cdot \phi_{\mathrm{pp}}\right)$ are the fourier components respectively of $f$ and $p_{r}$ at the perturbation frequency $f_{p}$. Once the response of $F R(s)$ is measured, it can be used to design controller $B(s)$, shown in Fig. 2a), for frequency support by the battery.

For the measurements of $F R(s)$ presented in this paper, the PLL in Fig. $2 \mathrm{~b}$ ) is designed with a $20-\mathrm{Hz}$ bandwidth. The amplitude of the injected active power perturbation, $\hat{P}_{p}$, is kept below $2 \mathrm{MW}$ to ensure a small-signal condition. The steady-state active power output of the battery, $P_{0}$, is kept zero. Table I summarizes the ratings and outputs of conventional generators for which $F R(s)$ is measured. Table I also lists the inertia and nominal droop gain of each generator. The active power output of wind and PV generators are shown in Fig. 1. Note that the total power supplied by the conventional and renewable generation for the considered operation condition is, respectively, 245.29 MW and $80 \mathrm{MW}$.

\section{EStIMATION OF FREQUENCY RESPONSE ChaRACTERISTICS}

\section{A. Primary Frequency Response}

Fig. 3 shows the response of $F R(s)$ for three different droop settings in the steam generators in Fig. 1. The droop settings of the hydro plants are kept unchanged at 0.05 . The magnitude response in Fig. 3 has the unit of $\mathrm{mHz} / \mathrm{MW}$. Fig. 3 shows that the droop setting of generators mainly affect the low-frequency gain of $F R(s)$. This is expected because the droop gains determine the quasi-steady-state frequency of the system following a transient event. The dc gains of the transfer function in Fig. 3 are 28.85 $\mathrm{mHz} / \mathrm{MW}(29.2 \mathrm{~dB}), 20.64 \mathrm{mHz} / \mathrm{MW}(26.3 \mathrm{~dB})$, and $10 \mathrm{mHz} /$ MW (20 dB), respectively, for the droop settings of $20 \%, 10 \%$, and $5 \%$ in the steam generators. Typically, the PFR is measured in a)

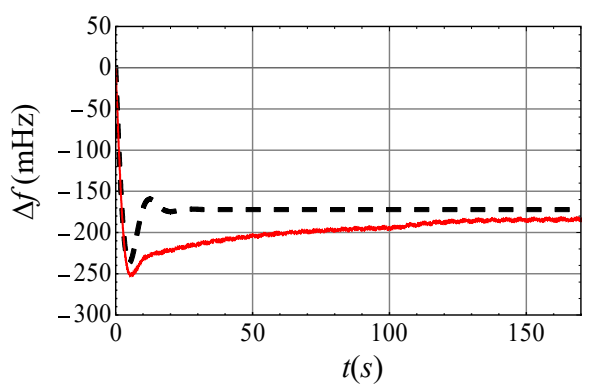

b)

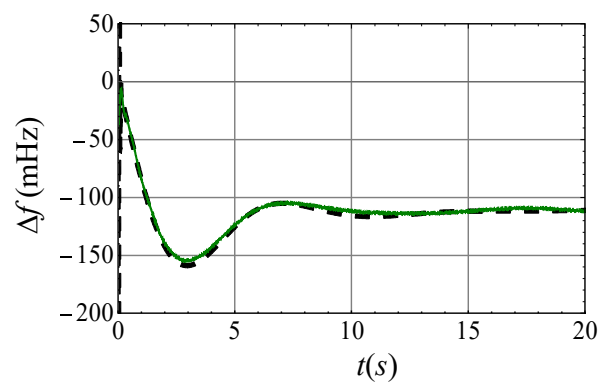

c)

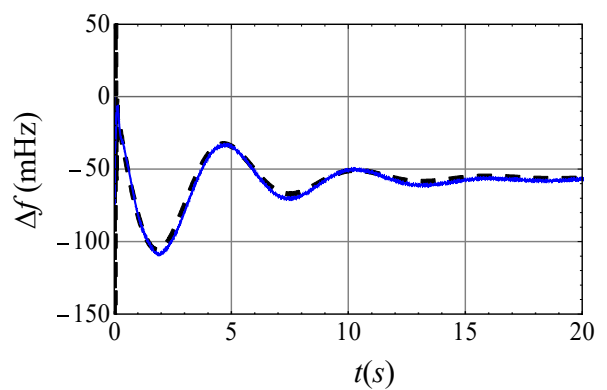

Fig. 4. Prediction of frequency response following a loss of generation event using frequency response transfer function $F R(s)$. Solid lines show simulated responses and dashed lines represent prediction by $F R(s)$. a) $20 \%$ droop, b) $10 \%$ droop, and c) $5 \%$ droop in the steam generators of the IEEE 9-bus system.

MW/0.1 Hz [2], which can be obtained directly by inverting the dc gains of $F R(s)$. The PFR predicted by $F R(s)$ is verified by simulating a generation loss event and comparing the steady-state frequency observed in simulations following the event with that predicted by the dc gain of $F R(s)$ from Fig. 3. Fig. 4 compares for different droop settings the frequency response obtained using dynamic simulations against those predicted by $F R(s)$ during the loss of a generator at Bus 1, which was supplying 5.7 MW before the fault. The frequency responses using $F R(s)$ in Fig. 4 are obtained using the step response of $F R(s)$ in Fig. 3 . The dc gain of $F R(s)$ in Fig. 3 predicts the drop in system frequency after the loss of a generation of $5.7 \mathrm{MW}$ to be $165 \mathrm{mHz}$ (20\% droop), $118 \mathrm{mHz}$ ( $10 \%$ droop), and $57 \mathrm{mHz}$ ( $5 \%$ droop). Fig. 4 shows that $F R(s)$ not only accurately predicts the PFR, but it also accurately predicts the frequency nadir and ROCOF following the event. Note that the slower recovery of frequency for the case of $20 \%$ droop in Fig. 4 as compared to the prediction by $F R(s)$ is because of the nonlinearities in the generator governors not captured by the response of $F R(s)$. This aspect will be studied further in a future work.

\section{B. System Inertia}

The resonance dipping in the response of $F R(s)$ in Fig. 3 at around $0.45 \mathrm{~Hz}$ is because of the series resonance between the capacitive behavior caused by the system inertia and the inductive 
behavior of the network transmission lines. The capacitance representing the system inertia is denoted by $C_{H}$, where $H$ signifies inertia constant. Based on the responses of $F R(s)$ in Fig. 3, the value of $C_{H}$ is estimated to be $0.065 \mathrm{~F}$.

If the effect of only the system inertia is considered, the active power and frequency can be related as:

$$
C_{H} \cdot \frac{d f}{d t}=\Delta P
$$

where $f$ is in $\mathrm{mHz}$ and $\Delta P$ is in MW. Based on (4), the measurements of $F R(s)$ predict the ROCOF to be $(0.065)^{-1}$, i.e., 15.38 $\mathrm{mHz} / \mathrm{s} / \mathrm{MW}$. This prediction is verified in the following by computing system inertia based on the inertia of generators.

The power system inertia equation can be written as:

$$
\frac{1}{60 \cdot 1000} \cdot \frac{d f}{d t}=\frac{1}{2 H_{\mathrm{sys}}} \frac{\Delta P}{S_{\mathrm{sys}}}
$$

where the gain $1 /(60 \cdot 1000)$ accounts for the conversion between the p.u. value of frequency to $\mathrm{mHz}, H_{\text {sys }}$ is the system inertia in seconds, $S_{\text {sys }}$ is the MVA rating of the system, and $\Delta P$ is the power disturbance in MW. As shown in Table I, the equivalent inertia of the system, $H_{\mathrm{sys}}$, is calculated to be $4.148 \mathrm{~s}$ depending on the inertia of the conventional generators in the system. Using the value of $H_{\mathrm{sys}}$ in (5) and comparing (5) with (4), the value of $C_{H}$ is obtained as 0.0658 . This matches the prediction using the responses of $F R(s)$. Hence, the response of $F R(s)$ accurately predicts system inertia and ROCOF.

\section{Speed of Primary Frequency Control}

Not only the PFR but also its speed is important for achieving desired frequency response following a transient event. The speed of the primary frequency control can be estimated by $F R(s)$. This is demonstrated by leveraging the fact that the primary frequency control of the hydro generators is typically much slower than that of the steam generators [6].

The behavior of the hydro generators in Fig. 1 is dominated by the 150-MVA generator at Bus 7 because its capacity is much higher than the other 20-MVA hydro generator at Bus 5 . However, the hydro generator at Bus 7 does not participate in the primary frequency control because of its operation outside the controllable range, which is the result of a lower limit of 0.4 p.u. on the gate position. To demonstrate how the speed of primary frequency control modifies $F R(s)$, the lower limit of the gate position of hydro generators is reduced to $0.1 \mathrm{p}$.u. This allows the 150-MVA hydro generator to participate in the primary frequency control of the system. Fig. 5 compares the response of $F R(s)$ before and after the change in the lower limit. It is evident that the participation of the 150-MVA hydro generator in primary frequency control has reduced the dc gain to $20 \mathrm{~dB}$ i.e. $10 \mathrm{mHz} /$ MW. The PFR of $10 \mathrm{mHz} / \mathrm{MW}$ is the same as the one that is obtained by reducing the droop gain of the steam generators from $20 \%$ to $5 \%$ (ref. Fig. 3). Hence, it can be expected that both cases - (a) reducing the droop gain of steam generators to $5 \%$ from $20 \%$ and (b) reducing the gate position lower limit of the hydro generators - will result in the same settling frequency after an event. Nonetheless, note from Fig. 5 that the PFR because of the hydro
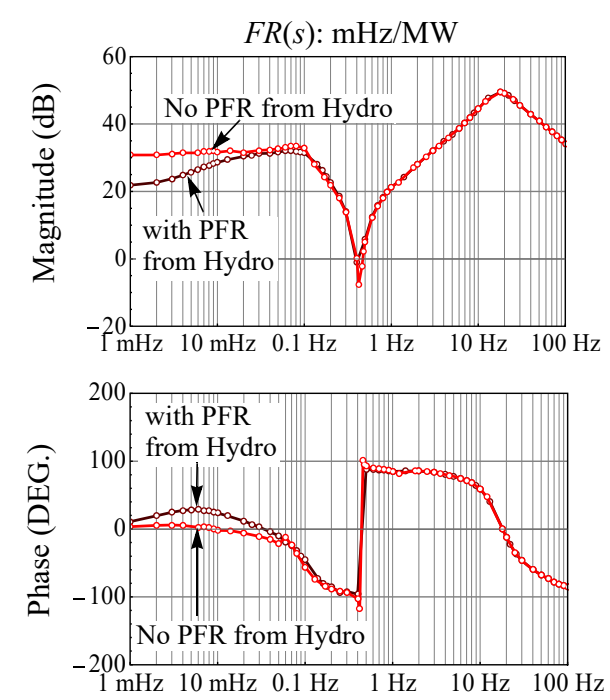

Fig. 5. Effect of the primary frequency control by hydro generators on the frequency response transfer function $F R(s)$.

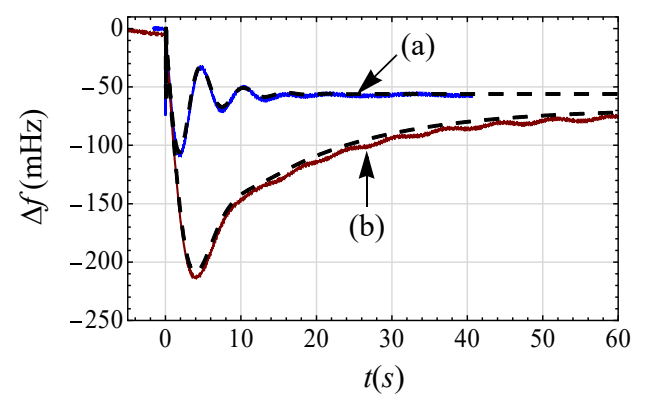

Fig. 6. Influence of the speed of primary frequency control: (a) thermal power plants operate with 5\% droop and no contribution from hydro generators, (b): thermal power plants operate with $20 \%$ droop and hydro generators operate with $5 \%$ droop. Solid lines show simulated responses and dashed lines show prediction by frequency response function $F R(s)$.

generators is much slower than that of the steam generators: the $\mathrm{dc}$ gain of $F R(s)$ is realized at around $1 \mathrm{mHz}$ in Fig. 5, when hydro generator is contributing to the PFR; whereas it is $10 \mathrm{mHz}$ when most of the PFR is coming from the steam generators. This shows that the PFR from the hydro generators is around 10 times slower than the thermal generators. This behavior is also confirmed in Fig. 6, which shows the frequency response after the loss of 5.7 MW of generation at Bus 1 when the entire PFR is coming from the steam generators and when the 150-MVA hydro generator also contributes to the PFR. In both cases, the system frequency settles to almost the same value because of the same amount of PFR of $10 \mathrm{mHz} / \mathrm{MW}$; however, the settling time is much longer and the frequency nadir is much worse when the PFR is contributed partially by the 150-MVA hydro generator.

\section{RELATION WITH IMPEDANCE}

The flow of the sinusoidal perturbation from $p_{r}$ to $f$ in Fig. 2a) can be described using the harmonic signal-flow graph shown in Fig. 7 [8]. It is drawn based on Fig. 2a) and the relationship between the frequencies of the perturbation component in $\mathrm{dc}$ and three-phase ac variables [8]. Note that the perturbation frequency 


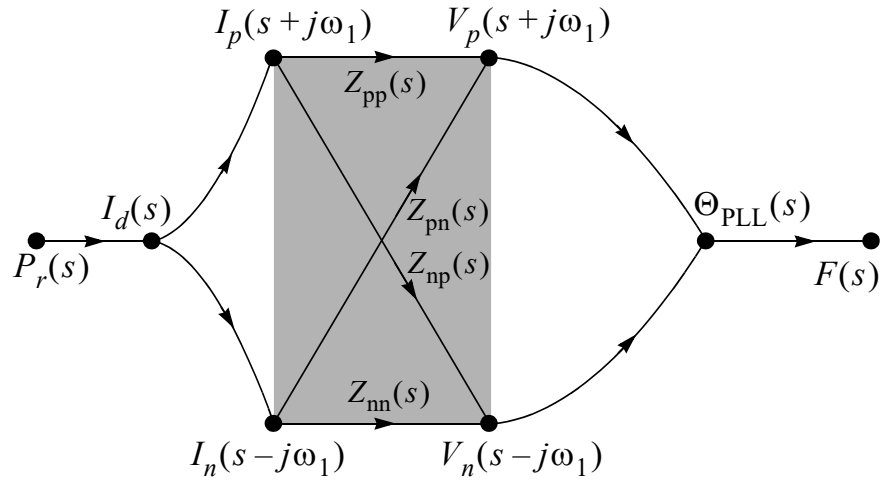

Fig. 7. Harmonic signal-flow graph showing the flow of perturbation from $p_{r}$ to $f$ in Fig. 2 for relating the frequency response transfer function $F R(s)$ with the network impedance. Note: $s=j 2 \pi f_{p}$ and $\omega_{1}=2 \pi f_{1}$.

of each node in Fig. 7 is identified in the parenthesis. The sequence of the perturbation components in $v_{\mathrm{abc}}$ and $i_{\mathrm{abc}}$ is identified by subscript $p$ or $n$, respectively, for the positive and negative sequence. For example, $I_{p}\left(s+j \omega_{1}\right)$ and $I_{n}\left(s-j \omega_{1}\right)$ in Fig. 7 represent, respectively, the positive sequence component at $f_{p}+f_{1}$ and the negative sequence component at $f_{p}-f_{1}$ in $i_{\text {abc }}$ in Fig. 2. Note that the branches in the shaded box represent the elements of the sequence-domain transfer matrix impedance $\boldsymbol{Z}_{\text {net }}(s)$ [9] of the network as seen from the point of interconnection.

Using the harmonic linearization method for developing the gains of branches in Fig. 7 based on the block diagram in Fig. 2a), the frequency response transfer function $F R(s)$ is obtained as:

$$
\begin{aligned}
F R(s) \equiv \frac{F(s)}{P_{r}(s)}= & -\frac{s}{2 \pi} \frac{j}{V_{i}} T_{\mathrm{PLL}}(s) \frac{1}{1+T_{i} s} \frac{2}{3 V_{1}} . \\
& \frac{1}{2}\left[Z_{\mathrm{pp}}(s)-Z_{\mathrm{np}}(s)+Z_{\mathrm{pn}}(s)-Z_{\mathrm{nn}}(s)\right]
\end{aligned}
$$

where $T_{\mathrm{PLL}}(s)=\left[V_{1} \cdot H_{\mathrm{PLL}}(s) / s\right] \cdot\left[1+V_{1} \cdot H_{\mathrm{PLL}}(s) / s\right]^{-1}$ is the closed-loop gain of the PLL; it can be approximated by unity below the PLL bandwidth of $20 \mathrm{~Hz}$.

Eq. (6) can be simplified by using the relationship between the sequence and dq domain impedances from [9] as:

$$
\begin{aligned}
F R(s) & =\frac{3}{2 V_{1}} \cdot \frac{s}{2 \pi} \cdot T_{\mathrm{PLL}}(s) \cdot \frac{1}{1+T_{i} s} \cdot \frac{1}{V_{1}} \cdot Z_{\mathrm{qd}}(s) \\
& \approx \frac{3}{2 V_{1}} \cdot \frac{s}{2 \pi} \cdot \frac{1}{V_{1}} \cdot Z_{\mathrm{qd}}(s) \quad \text { for } f<\text { PLL BW }
\end{aligned}
$$

where:

- $3 /\left(2 V_{1}\right)$ represents the gain from the perturbation in the active power reference, $p_{r}$, to the perturbation in the daxis current reference, $i_{\mathrm{dr}}$

- $s /(2 \pi)$ converts the perturbation in the angle of the PCC voltages $v_{\mathrm{abc}}$ in radians to frequency in $\mathrm{Hz}$;

- $1 / V_{1}$ represents the gain from the perturbation in the qaxis component, $v_{q}$, to the perturbation in the angle of the PCC voltages, $v_{\mathrm{abc}}$;

- $Z_{\mathrm{qd}}(s)$ is an element of the dq-domain impedance of the network that relates the d-axis component of the BESS output currents, $i_{\mathrm{abc}}$, to the q-axis component of the PCC voltages, $v_{\mathrm{abc}}$.

It is interesting to note from (7) that the frequency response function, $F R(s)$, is shaped by $Z_{\mathrm{qd}}(s)$ elements of the dq-domain impedance of the network. This intuitively makes sense because the perturbation in $i_{d}$ is proportional to the perturbation in the active power input to the network and the perturbation in $v_{q}$ is proportional to the perturbation in the angle of $v_{\mathrm{abc}}$ [9].

Eq. (7) shows that the $Z_{\mathrm{qd}}(s)$ element of the dq-domain impedance of a network, a generator, or an inverter captures its frequency response behavior. Hence, the response of $Z_{\mathrm{qd}}(s)$ can be used to estimate the contribution of an inverter to the inertia and PFR of a power system.

\section{CONCLUSIONS}

This paper presented an impedance-based noninvasive approach for real-time characterization of power system frequency response in the absence of a transient event. It showed that the transfer function from the active power injected at the point of interconnection to the frequency at the same bus can be used to estimate system inertia, primary frequency response, and the speed of the primary frequency control. The paper also developed relationship between the so-called frequency response transfer function and the network impedance as seen from the point of interconnection; such relationship can support the development of grid-friendly controls for inverters for simultaneously optimizing dynamic stability and frequency adequacy of the system. Future work will evaluate the selection of the active power perturbation magnitude depending on system size and characteristics, as well as measurement noise. An equivalent frequency-domain approach for the characterization of voltage response of a power system will also be developed.

\section{REFERENCES}

[1] "State of reliability," NERC, Atlanta, GA, USA, June 2018.

[2] V. Gevorgian, Y. Zhang, and E. Ela, "Investigating the impacts of wind generation participation in interconnection frequency response," IEEE Trans. Sustain. Energy, vol. 6, no. 3, pp. 1004-1012, July 2015.

[3] J. W. Ingleson and D. M. Ellis, "Tracking the eastern interconnection frequency governing characteristics," in Proc. 2005 IEEE Power Eng. Soc. General Meeting, San Francisco, CA, June 2005.

[4] P. M. Ashton, et. al., "Inertia estimation of the GB power system using synchrophasor measurements," IEEE Trans. Power Syst., vol. 30, no. 2, pp. 701-709, Mar. 2015.

[5] Federal Energy Regulatory Commission, "BAL-003-1-Frequency response and frequency bias setting," Standard, Washington, DC, USA, Mar. 2013.

[6] J. Undrill, "Primary frequency response and control of power system frequency," Lawrence Berkeley National Laboratory, CA, USA, Rep. LBNL-2001105, Feb. 2018.

[7] F. Wilches-Bernal, J. H. Chow, and J. J. Sanchez-Gasca, "A fundamental study of applying wind turbines for power system frequency support," IEEE Trans. Power Syst., vol. 31, no. 2, pp. 1496-1505, Mar. 2016.

[8] S. Shah and L. Parsa, "Small-signal modeling of single-phase PLLs using harmonic signal-flow graphs," in Proc. 2017 IEEE Energy Conv. Cong \& Expo., Cincinnati, OH, USA, 2017, p. 7.

[9] S. Shah and L. Parsa, "Impedance modeling of three-phase voltage source converters in dq, sequence, and phasor domains," IEEE Trans. Energy Conv., vol. 32, no. 3, pp. 1139-1150, Sep. 2017. 